\title{
The promise of neuroscience for understanding the cultural shaping of emotion and other feelings
}

\author{
Jeanne L. Tsai ${ }^{1}$ Yang $\mathbf{Q u}^{2}$ \\ Published online: 2 August 2018 \\ (C) Springer-Verlag GmbH Germany, part of Springer Nature 2018
}

Decades of research in psychology, anthropology, and sociology demonstrate cultural differences in people's emotions and other feelings, ranging from what people believe about their emotions, to how they express them and perceive them in others, to how they control and regulate them. However, given evidence for cultural similarities in emotion, questions remain unanswered regarding how deeply cultural differences "get under the skin," and what neural mechanisms support them. Until recent methodological developments in neuroscience, researchers were largely unable to answer these questions. For instance, the development of functional magnetic resonance imaging (fMRI) and electroencephalography (EEG) allows researchers to examine whether cultural differences in emotion self-reports and behavior are also reflected in activity in specific areas of the brain related to reward, attention, and self-relevance. Similarly, advances in genetic methods not only allow researchers to assess whether cultural differences in emotional experience are linked to differences in genetic expression, but also to examine how cultural and genetic influences interact (for reviews of previous work, see Chiao and Ambady 2007; Han and Northoff 2008; Han et al. 2013; Kitayama and Uskul 2011).

In this special issue, we present five papers that employ these new methods to address the cultural shaping of emotion perception, emotional experience, and emotion regulation. Park et al. (2018a) use fMRI to examine the neural mechanisms underlying cultural differences in affiliative judgments of excited versus calm targets, and find that these differences are related to reward processes. Lin et al. (2018) use fMRI to examine the neural mechanisms underlying cultural differences in perceptions of racial ingroup and outgroup members, and find that these differences are related to visual attention processes. Park et al. (2018b) use EEG to investigate why Asian Americans typically report higher social anxiety than other groups, and find that increases in social anxiety may be related to less visual

Jeanne L. Tsai

jltsai@stanford.edu

$凶$ Yang Qu

yangqu@northwestern.edu

1 Stanford University, Stanford, CA, USA

2 School of Education and Social Policy, Northwestern University, Evanston, IL, USA 
processing of specific emotional expressions. Tompson et al. (2018) demonstrate how genetic variation within cultures may explain why some individuals are better able to emotionally achieve their culture's ideal more than others. Finally, Hampton and Varnum (2018) summarize what we know and still need to know about emotion regulation in different cultures based on studies that use various neuroscience methods, including their own.

Together, these studies suggest that cultural differences in emotional self-reports and behavior run deep, are associated with specific neural mechanisms related to reward and attention, and may be partly related to genetic factors. However, at the same time that these papers provide deeper understandings of cultural differences, they raise additional challenges that need to be addressed as researchers continue to integrate neuroscience methods into their work. The first challenge is creating emotionally evocative stimuli and tasks that simulate what happens in people's daily lives and that also allow collection of neural and genetic data. The second challenge is integrating cultural patterns that may vary at different levels of analysis; for example, how do we interpret cultural differences in self-report and behavior that may be inconsistent with cultural differences in brain activity? And the third is establishing causality - do cultural differences in brain activity give rise to cultural differences in behavior; is the reverse true; or are there bidirectional influences? These studies also demonstrate the importance of reliable and valid measures of the cultural ideas and practices of interest that can be linked to neuroscience data, and the need for studies that build on but also move beyond comparisons of US and East Asian contexts. Only then can the full promise of neuroscience methods for understanding the cultural shaping of emotion be realized.

\section{References}

Chiao, J. Y., \& Ambady, N. (2007). Cultural neuroscience: Parsing universality and diversity across levels of analysis. In S. Kitayama \& D. Cohen (Eds.), Handbook of cultural psychology (pp. 237-254). New York, NY: Guilford Press.

Hampton, R. S., \& Varnum, M. E. W. (2018). The cultural neuroscience of emotion regulation. Culture and Brain. https://doi.org/10.1007/s40167-018-0066-2.

Han, S., \& Northoff, G. (2008). Culture-sensitive neural substrates of human cognition: A transcultural neuroimaging approach. Nature Reviews Neuroscience, 9(8), 646-654. https://doi.org/10.1038/ nrn2456.

Han, S., Northoff, G., Vogeley, K., Wexler, B. E., Kitayama, S., \& Varnum, M. E. W. (2013). A cultural neuroscience approach to the biosocial nature of the human brain. Annual Review of Psychology, 64, 335-359. https://doi.org/10.1146/annurev-psych-071112-054629.

Kitayama, S., \& Uskul, A. K. (2011). Culture, mind, and the brain: Current evidence and future directions. Annual Review of Psychology, 62, 419-449. https://doi.org/10.1146/annurev-psych120709-145357.

Lin, L. C., Qu, Y., \& Telzer, E. H. (2018). Cultural influences on the neural correlates of intergroup perception. Culture and Brain. https://doi.org/10.1007/s40167-018-0070-6.

Park, B., Qu, Y., Chim, L., Blevins, E., Knutson, B., \& Tsai, J. L. (2018a). Ventral striatal activity mediates cultural differences in affiliative judgments of smiles. Culture and Brain. https://doi.org/ 10.1007/s40167-018-0061-7.

Park, G., Lewis, R. S., Wang, Y. C., Cho, H. J., \& Goto, S. G. (2018b). Are you mad at me? Social anxiety and early visual processing of anger and gaze among Asian American biculturals. Culture and Brain. https://doi.org/10.1007/s40167-018-0067-1. 
Tompson, S. H., Huff, S. T., Yoon, C., King, A., Liberzon, I., \& Kitayama, S. (2018). The dopamine D4 receptor gene (DRD4) modulates cultural variation in emotional experience. Culture and Brain. https://doi.org/10.1007/s40167-018-0063-5. 